\title{
Infection Control Impact on Patient with Tuberculosis Chroretinitis in KSA Hospital Based Analysis
}

\author{
Mohammed Ahmed Garout ${ }^{1}$, Emad Algahdaly ${ }^{1}$, Magda Ramadan Abdultawab ${ }^{2, *}$, \\ Rana Mohammed Garout ${ }^{3}$, Raha Mohammed Garout ${ }^{3}$ \\ ${ }^{1}$ Community Medicine \& Public Health, Umo Alqurah University, Faculty of Medicine, Makkah, KSA \\ ${ }^{2}$ Microbiology \& Immunology \& Infection Control, Faculty of Medicine, Ainshams University, Cairo, Egypt \\ ${ }^{3}$ Medical Student, IbnSina National Collage for Medical Studies, Jeddah, KSA
}

Email address:

Ramadan_magda@yahoo.com (M. R. Abdultawab)

*Corresponding author

\section{To cite this article:}

Mohammed Ahmed Garout, Emad Algahdaly, Magda Ramadan Abdultawab, Rana Mohammed Garout, Raha Mohammed Garout. Infection Control Impact on Patient with Tuberculosis Chroretinitis in KSA Hospital Based Analysis. American Journal of Internal Medicine. Vol. 7, No. 3, 2019, pp. 77-80. doi: 10.11648/j.ajim.20190703.15

Received: May 8, 2019; Accepted: June 14, 2019; Published: June 29, 2019

\begin{abstract}
Tuberculosis (TB) is an infectious disease responsible for significant morbidity and mortality worldwide. WHO estimates that one third of the world's population is currently infected, with 9 million new cases occurring annually, leading to 3 million deaths per year, the disease affects the ocular anterior segment, the posterior segment, and adnexa., The intraocular manifestations of TB include "mutton fat" keratic precipitates, posterior synechiae, vitreous snowballs, snow banking, retinal vasculitis, choroiditis, serpiginous-like choroiditis, and panuveitis] A definitive diagnosis of intraocular TB requires culture data, and direct demonstration of Mycobacterium tuberculosis in smears and via polymerase chain reaction (PCR) of intraocular fluid, The purpose of this study is to describe the ocular manifestations, diagnosis and treatment of tuberculosis and to emphasize the fact that ocular tuberculosis may occur in the absence of systemic clinical activity and may mimic several clinical entities The current study is an observational consecutive case series of patients diagnosed with intraocular tuberculosis managed at a hospital. Study the prevalence of ocular TB in cross section eye care center Magrabi hospital Jeddah, KSA, from June 1, 2013 to December 31, 2015. diagnosis of ocular TB is often presumed in the presence of suggestive ocular findings in combination with any of the following: Systemic findings consistent with TB infection, Positive interferon gamma release assay, Positive tuberculin skin test in asymptomatic individuals, Acid-fast bacilli (AFB) on direct smear or culture of MTB from ocular samples, Polymerase chain reaction (PCR), Fluorescein angiography (FA), RESULTS: 86 of 2542 patients for examination of retina in retina clinic were diagnosed as suspected tuberculosis chroretinitis, 20 percent of them had conformed diagnosed as TB. out Of 86, $24(27.9 \%)$ had at least one episode of oculacomplaints, 7 patients were conformed with PCR. CONCLUSION: The diagnosis of presumed ocular tuberculosis remains a clinical challenge, although interferon- $\gamma$ release assays they currently lack the specificity to distinguish between latent tuberculosis infection and active tuberculosis. molecular diagnostic PCR may be valuable in our ability to establish an earlier etiologic diagnosis and institute appropriate antimycobacterial therapy and ensure from implementation of infection control measurements.
\end{abstract}

Keywords: Mycobacterium Tuberculosis, Choroidal Granuloma, Retinal Vasculitis, PCR, Acid-fast Bacilli (AFB)

\section{Introduction}

\subsection{Literature Review}

Tuberculosis (TB) is one of the leading public health problems in developing countries. Diagnosis of isolated ocular TB in the absence of systemic TB and other ocular findings is important, The intraocular manifestations of TB include "mutton fat" keratic precipitates, posterior synechiae, vitreous snowballs, snowbanking, retinal vasculitis, choroiditis, serpiginous-like choroiditis, and panuveitis.[1] A definitive diagnosis of intraocular TB requires culture data, 
and direct demonstration of Mycobacterium tuberculosis in smears and via polymerase chain reaction (PCR) of intraocular fluid. The frequencies of ocular TB in patients with uveitis and systemic TB are respectively $0-0.16 \%$ and $0.27-1.4 \%$. [1]

Tuberculosis (TB) is an infection caused by Mycobacterium tuberculosis, which can cause disease in multiple organs throughout the body, including the eye. The term "ocular TB" describes an infection by the M. tuberculosis species that can affect any part of the eye (intraocular, superficial, or surrounding the eye. [2]

TB is an airborne communicable disease that most commonly involves the lungs. Nearly one-third of the world's population is latently infected with $\mathrm{TB}$, and more than 9 million new cases are diagnosed each year, 95\% in developing countries. Ocular TB may not be associated with clinical evidence of pulmonary $\mathrm{TB}$; up to $60 \%$ of patients with evidence of extrapulmonary TB may not have diagnosed pulmonary TB, Ocular TB may be an initial presentation of extrapulmonary dissemination of infection; Posterior uveitis is the most common presentation of intraocular TB. [3]

Ocular involvement from TB can either occur as a primary active infection or a secondary infection from a distant site, usually through hematogenous spread. The conjunctiva, cornea, and sclera are sites of primary ocular involvement. Hematogenous spread is the primary mechanism by which TB affects the eye [4].

\subsection{Objective of Study}

The purpose of this review is to describe the ocular manifestations, diagnosis and treatment of tuberculosis and to emphasize the fact that ocular tuberculosis may occur in the absence of systemicclinical activity and may mimic several clinical entities. The current study is an observational consecutive case series of patients diagnosed with intraocular tuberculosis managed at a hospital.

\section{Study Methods}

i. To study the prevalence of ocular TB in cross section eye care centre Magrabi hospital Jeddah, KSA, from June 1, 2013 to December 31, 2015.

This was a cross-sectional descriptive study approved by magrabi eye hospital board analyze the clinical profiles, histopathologic features, and Mycobacterium tuberculosis polymerase chain reaction testing in patients with ocular tuberculosis. Clinical manifestations of ocular TB vary, which poses a challenge for diagnosis. The definitive diagnosis of TB is established by isolation of $M$ tuberculosis bacilli from ocular tissues. However, because this is difficult to achieve, the diagnosis of ocular TB is often presumed in the presence of suggestive ocular findings in combination with any of the following: Systemic findings consistent with TB infection.

Positive interferon gamma release assay, Positive tuberculin skin test in asymptomatic individuals:

ii. Microbiological or histopathological evidence of $M$ tuberculosis: Acid-fast bacilli (AFB) on direct smear or culture of MTB from ocular samples gold standard, but it is low sensitivity, delayed diagnosis due to slowgrowing nature of bacteria.

iii. Tuberculin skin testing (TST) Used to assess immunological evidence of MTB infection, latent or active. low cost and easy availability, but it is subjective, low sensitivity and specificity, potential false- positive in prior vaccinated patients, falsenegatives in immunocompromised.

iv. Interferon-gamma release assays (IGRA) (Quantiferon Gold) blood test that quantifies IFN-gamma more specific, but does not distinguish between latent and active disease Polymerase chain reaction (PCR) rapidly detecting mycobacterial genome from small sample of ocular fluid; allows fast detection and quantifies pathogen load in sample, also be helpful in identifying drug-resistant strains of Mycobacterium.

v. Chest X-ray (CXR) To evaluate patients with suspected intraocular $\mathrm{TB}$, when lungs are primary site of $\mathrm{TB}$ infection.

vi. Fluorescein angiography (FA) evaluation of retinal vascular leakage and active choroidal lesions, Ultrasonograph of large tuberculomas.

vii.Patients diagnosed with active TB should also be tested for HIV due to high incidence of co-infection.

\section{Results}

During January 2013-december 2015, 86 of 2542 patients for examination of retina in retina clinic were diagnosed as suspected tuberculosis chroretinitis, patients with uveitis were treated at a Magrabi Eye Hospital care, The median age of these 86 patients was 25 (20-35) years and $47 \%$ were males. 20 percent of them had conformed diagnosed as TB. out of $86,24(27.9 \%)$ had at least one episode of ocular complaints (the majority blurred-vision) and among them, five $(5.8 \%)$ had optic neuropathy. Patients received appropriate treatment and improvements were observed. 7 patients were conformed with PCR rifampicin resistance.

Table 1. Prevalence of ocular TB in Region-Specific Information.

\begin{tabular}{ll}
\hline Country & Prevalence of presumed ocular TB \\
\hline India & $0.4-9.8 \%$ \\
China & $4.0 \%$ \\
Japan & $7.0 \%$ \\
Thailand & $2.2 \%$ \\
Philippines & $6.8 \%$ \\
\hline
\end{tabular}


Table 2. Prevalence of ocular TB.

\begin{tabular}{ll}
\hline Items & Results \\
\hline Number of patients enter in the study & 2542 \\
Number of patients with ocular tuberculosis & 86 \\
Percentage & 3.38 \\
\hline
\end{tabular}

Table 3. Demographics data and symptoms of ocular TB.

\begin{tabular}{ll}
\hline Items & Prevalence of presumed ocular TB \\
\hline Age & Mean age 25 (20-35) years \\
Sex percentage & $47 \%$ were males \\
conformed diagnosed as TB & 20 percentage \\
one episode of ocular complaints (the majority blurred-vision) & $24(27.9 \%)$ \\
optic neuropathy & five $(5.8 \%)$ \\
conformed with PCR rifampicin resistance & 7 patients \\
\hline
\end{tabular}

\section{Discussion}

TB may affect the eye by direct invasion of the tubercle bacillus following hematogenous dissemination with local destruction and inflammation, or via a hypersensitivity reaction to the bacillus located elsewhere in the body. Ocular TB has several potential manifestations. It can appear on the external eye as a lid abscess or manifest as chronic blepharitis or atypical chalazions. It can present as amucopurulent conjunctivitis with regional lymphadenopathy. It can also present as a phlyctenule (an inflammatory nodule at the junction of the cornea and sclera), infectious keratitis, inflammation within the cornea stroma (interstitial keratitis), or as an infectious scleritis. All of these presentations are rare and are easy to diagnose as material can be obtained for culture and biopsy. Rarely, orbital disease can also occur. $[5,6]$

Preventing TB transmission in healthcare facilities requires early identification, isolation, and treatment of patients with active TB disease. Recommended infection control strategies to reduce TB transmission depend on the prevalence of active $\mathrm{TB}$ in the patient population and the resources available to implement control programs. Administrative controls are the first and most important level of TB control in healthcare settings. The following measures should be taken: Assign responsibility to an infection control officer for implementation, enforcement, and evaluation of TB infection control policies. Conduct a TB risk assessment at the facility to include identifying the number of TB patients seen at the facility, the amount of time TB patients spend in different areas (such as emergency rooms, waiting rooms or wards), Develop and implement a TB infection control policy to ensure prompt detection, isolation, and treatment of persons with suspected or confirmed TB disease and education for healthcare workers as adherence to TB control measures, Ensure proper cleaning and disinfection of potentially contaminated equipment, Continue airborne isolation of patients with active TB until they are no longer infectious. [7]

Also all patient attend as outpatient clinic \&no patient admitted as active tuberculosis under airborne isolation Ocular involvement from TB can either occur as a primary active infection or a secondary infection from a distant site, usually through hematogenous spread. The conjunctiva, cornea, and sclera are sites of primary ocular involvement. Table 1 prevalence of ocular TB in different country, our study near the same as prevalence of ocular TB in eye hospital in Saudi Arabia were 3.38 percentage (table 2).

Tuberculous uveitis is a vision-threatening disease that inevitably leads to blindness if not properly diagnosed and treated. Which appeared in our study table 3 The diagnosis of ocular TB is presumptive, and it is unknown if ocular manifestations result from a delayed hypersensitivity reaction or due to the infectious agents [8]. This is reflected in the absence of information on ocular TB management in any of the TB guidelines of the UK, USA, or Canada [9]

Our study revealed that Patients received appropriate treatment and improvements were observed.

Multidrug-resistant TB (MDR-TB) remains a public health crisis and a health security threat. WHO estimates that there were 558000 new cases with resistance to rifampicin - the most effective first-line drug, of which - $82 \%$ had MDR-TB.

Globally, TB incidence is falling at about $2 \%$ per year. This needs to accelerate to a $4-5 \%$ annual decline to reach the 2020 milestones of the End TB Strategy [10].

Olivier etal; 2018 found that the incidence of pulmonary tuberculosis was significantly lower with M72/AS01E than with placebo among healthy $M$. tuberculosis-infected, largely BCG-vaccinated, HIV-negative adults. These promising results provide an opportunity to better understand the mechanisms by which this vaccine may confer protection against tuberculosis and support its further evaluation [11].

The theme of World TB Day 2019 - 'It's time' - puts the accent on the urgency to act on the commitments made by global leaders to:

1. Scale up access to prevention and treatment;

2. Build accountability;

3. Ensure sufficient and sustainable financing including for research;

4. Promote an end to stigma and discrimination, and;

5. Promote an equitable, rights-based and people-centered TB response. [12]

\section{Conclusion}

The diagnosis of presumed ocular tuberculosis remains a 
clinical challenge, Although interferon- $\gamma$ release assays they currently lack the specificity to distinguish between latent tuberculosis infection and active tuberculosis. molecular diagnostic PCR may be valuable in our ability to establish an earlier etiologic diagnosis and institute appropriate antimycobacterial therapy and ensure from implementation of infection control measurements.

\section{Conflict of Interest}

No conflict of interest to declare.

\section{References}

[1] A Akal, T Goncu, FN Boyaci, ZHA Sak, F Yalcin, U Ozkan, and T Ulas: Primary Tubercular Chorioretinitis: Ann Med Health Sci Res. 2014 Nov-Dec; 4 (6): 965-967.

[2] Centers for Disease Control and Prevention: Racial disparities in Tuberculosis-selected southeastern states. 2004. MMWR Morb Mortal Wkly; Rep 53: 556-9.

[3] Sharma, B. Thapa, P. Lavaju: Ocular tuberculosis: an update, Nepal J Ophthalmol, 3 (5) (2011), pp. 52-67.

[4] Gupta, V. Gupta: Tubercular posterior uveitis, Int Ophthalmol Clin, 2005, pp. 71-88.

[5] Gonzalo G. Alvareza, Virginia R. Roth William Hodgeb: PlumX Metrics: Ocular tuberculosis: diagnostic and treatment challenges, 2009 Volume 13, Issue 4, Pages 432-435: https://doi.org/10.1016/j.ijid.2008.09.018.
[6] Yeh S, Sen HN, Colyer M, Zapor M, Wroblewski K: Curr Opin Ophthalmol. 2012 Nov; 23 (6): 551-6. Update on ocular tuberculosis.

[7] United States Center for Diseases Control (CDC) TB Treatment Guidelines: https://www.cdc.gov/tb/publications/guidelines/treatment.htm/ 2018.

[8] Rao NA, Saraswathy S, Smith RE. Tuberculous uveitis: distribution of mycobacterium tuberculosis in the retinal pigment epithelium. Arch Ophthalmol. 2006; 124: 1777-1779. [PubMed] [Google Scholar].

[9] Sanghvi C, Bell C, Woodhead M, Hardy C, Jones N. Presumed tuberculous uveitis: diagnosis, management, and outcome. Eye (Lond) 2011; 25: 475-480. [PMC free article] [PubMed] [Google Schola.

[10] WHO. 2018 https://www.who.int/news-room/factsheets/detail/tuberculosis.

[11] Olivier Van Der Meeren, M. D., Mark Hatherill, M. D., Videlis Nduba, M. B., Ch. B., M. P. H., Robert J. Wilkinson, F. Med. Sci., Monde Muyoyeta, M. B., Ch. B., Ph. D., Elana Van Brakel, M. B., Ch. B., Helen M. Ayles, M. B., B. S., Ph. D., German Henostroza, M. D: Phase 2b Controlled Trial of M72/AS01E Vaccine to Prevent Tuberculosis, 2018. New England Journal of Medicine September 25, 2018, N Engl J Med 2018; 379: 1621-1634 DOI: 10.1056/NEJMoa1803484.

[12] WHO: World Tuberculosis Day 2019. https://www.who.int/newsroom/events/detail/2019/03/24/default-calendar/world-tb-day2019. 